\title{
On the simplest quartic fields and related Thue equations
}

\author{
AKINARI Hoshi \\ Department of Mathematics, Rikkyo University \\ 3-34-1 Nishi-Ikebukuro Toshima-ku Tokyo, 171-8501, Japan \\ hoshi@rikkyo.ac.jp
}

\begin{abstract}
Let $K$ be a field of char $K \neq 2$. For $a \in K$, we give an explicit answer to the field isomorphism problem of the simplest quartic polynomial $X^{4}-a X^{3}-6 X^{2}+a X+1$ over $K$ as the special case of the field intersection problem via multi-resolvent polynomials. From this result, over an infinite field $K$, we see that the polynomial gives the same splitting field over $K$ for infinitely many values $a$ of $K$. We also see by Siegel's theorem for curves of genus zero that only finitely many algebraic integers $a \in \mathcal{O}_{K}$ in a number field $K$ may give the same splitting field. By applying the result over the field $\mathbb{Q}$ of rational numbers, we establish a correspondence between primitive solutions to the parametric family of quartic Thue equations
\end{abstract}

$$
X^{4}-m X^{3} Y-6 X^{2} Y^{2}+m X Y^{3}+Y^{4}=c,
$$

where $m \in \mathbb{Z}$ is a rational integer and $c$ is a divisor of $4\left(m^{2}+16\right)$, and isomorphism classes of the simplest quartic fields.

\section{Introduction and main results}

Let $K$ be a field of char $K \neq 2$ and $K(s)$ the rational function field over $K$ with variable $s$. We take the simplest quartic polynomial

$$
f_{s}(X):=X^{4}-s X^{3}-6 X^{2}+s X+1 \in K(s)[X]
$$

with discriminant $4\left(s^{2}+16\right)^{3}$. The Galois group $\operatorname{Gal}_{K(s)} f_{s}(X)$ of the polynomial $f_{s}(X)$ over $K(s)$ is isomorphic to the cyclic group $C_{4}$ of order four.

In the case where $K=\mathbb{Q}$, for $a \in \mathbb{Z} \backslash\{0, \pm 3\}$ the polynomials $f_{a}(X)$ are irreducible over $\mathbb{Q}$ with $\operatorname{Gal}_{\mathbb{Q}} f_{a}(X) \cong C_{4}$ and the splitting fields $\operatorname{Spl}_{\mathbb{Q}} f_{a}(X)$ of $f_{a}(X)$ over $\mathbb{Q}$ are totally real cyclic quartic number fields which are called the simplest quartic fields (cf. e.g. Gra77, [Laz91], LP95], Gaa02, Section 6.2], Kim04, [HH05], Duq07, Lou07]).

For $b=-a \in K$, the polynomials $f_{a}(X)$ and $f_{b}(X)$ have the same splitting field over $K$. In the present paper, we consider the field isomorphism problem of $f_{s}(X)$, i.e. for a fixed $a \in K$, determine whether $b \in K$ gives the same splitting field over $K$ as $\operatorname{Spl}_{K} f_{a}(X)=\operatorname{Spl}_{K} f_{b}(X)$ or not.

For $n \geq 3$, Rikuna Rik02 constructed one-parameter families of cyclic polynomials of degree $n$ over $K$ with char $K \backslash n$ and $K \ni \zeta+\zeta^{-1}$ where $\zeta$ is a primitive $n$-th root of unity, and $f_{s}(X)$ may be obtained the quartic case $n=4$ of Rikuna's cyclic polynomials (see also Miy99, [HM99]). An answer to the field isomorphism problem to Rikuna's cyclic

2000 Mathematics Subject Classification. Primary 11D25, 11D59, 11R16, 11Y40, 12 F10.

Key words and phrases. Field isomorphism problem, simplest quartic fields, quartic Thue equations, multiresolvent polynomial.

This work was partially supported by Rikkyo University Special Fund for Research. 
polynomials was given by Komatsu [Kom04] as a generalization of Kummer theory (cf. also Oga03, Kid05]).

In Section 3 , by using multi-resolvent polynomials, we will give an explicit form of an answer to the field isomorphism problem of $f_{s}(X)$ over $K$ as the special case of the field intersection problem (cf. the simplest cubic case [Mor94, Cha96, Oga03, Kom04, [HM09a], [H] ). One of the advantages of using multi-resolvent polynomials is the validity for non-abelian groups (see [HM07, [HM09b], [HM09c, [HM]).

Theorem 1.1. Let $K$ be a field of char $K \neq 2$ and $f_{a}(X)=X^{4}-a X^{3}-6 X^{2}+a X+1 \in K[X]$ for $a \in K$. For $a, b \in K$ with $a \neq \pm b$ and $\left(a^{2}+16\right)\left(b^{2}+16\right) \neq 0$, the following three conditions are equivalent:

(i) the splitting fields of $f_{a}(X)$ and of $f_{b}(X)$ over $K$ coincide;

(ii) the polynomial $f_{A}(X)$ splits completely into four linear factors over $K$ for $A=A_{1}$ or $A=A_{2}$ where

$$
A_{1}=\frac{a b+16}{-a+b} \text { and } A_{2}=\frac{a b-16}{a+b}
$$

(iii) there exists $z \in K$ such that

$$
B=a+\frac{\left(a^{2}+16\right) z(z+1)(z-1)}{f_{a}(z)}
$$

where $B=b$ or $B=-b$.

Moreover if $\operatorname{Gal}_{K} f_{a}(X) \cong C_{4}\left(\right.$ resp. $\operatorname{Gal}_{K} f_{a}(X) \cong C_{2}$ or $\left.\{1\}\right)$ then (ii) occurs for only one of $A_{1}$ and $A_{2}$ (resp. for both of $A_{1}$ and $A_{2}$ ) and (iii) occurs for only one of $b$ and $-b$ (resp. for both of $b$ and $-b)$.

Note that the equivalence of the conditions (i) and (iii) is valid also for $a= \pm b$.

By Theorem 1.1, for a fixed $a \in K$ with $a^{2}+16 \neq 0$, we have $\operatorname{Spl}_{K} f_{b}(X)=\operatorname{Spl}_{K} f_{a}(X)$ where $b$ is given as in Theorem 1.1 (iii) for arbitrary $z \in K$ with $f_{a}(z) \neq 0$ and $b^{2}+16 \neq 0$. Hence we have the following:

Corollary 1.2. Let $K$ be an infinite field of char $K \neq 2$. For a fixed $a \in K$ with $a^{2}+16 \neq 0$, there exist infinitely many $b \in K$ such that $\operatorname{Spl}_{K} f_{b}(X)=\operatorname{Spl}_{K} f_{a}(X)$.

The following theorem is well-known as Siegel's theorem for curves of genus zero (cf. [Lan78, Theorem 6.1], Lan83, Chapter 8, Section 5]).

Theorem (Siegel). Let $K$ be a number field and $\mathcal{O}_{K}$ the ring of integers in $K$. If a rational function $\varphi(s) \in K(s)$ has at least three distinct poles, then there are only finitely many $z \in K$ such that $\varphi(z) \in \mathcal{O}_{K}$.

In contrast with Corollary 1.2, by applying Siegel's theorem to Theorem 1.1, we get:

Corollary 1.3. Let $K$ be a number field and $\mathcal{O}_{K}$ the ring of integers in $K$. Assume that $a \in \mathcal{O}_{K}$ with $a^{2}+16 \neq 0$. Then there exist only finitely many integers $b \in \mathcal{O}_{K}$ such that $\operatorname{Spl}_{K} f_{b}(X)=\operatorname{Spl}_{K} f_{a}(X)$. In particular, there exist only finitely many integers $b \in \mathcal{O}_{K}$ such that $f_{A_{i}}(X),(i=1,2)$, has a linear factor over $\mathbb{Q}$ where $A_{i}$ is given in Theorem 1.1 (ii).

We treat the case of $K=\mathbb{Q}$ and $a=m \in \mathbb{Z}$. We get an application of Theorem 1.1 to a related family of quartic Thue equations as follows.

Consider the parametric family of quartic Thue equations

$$
F_{m}(X, Y):=X^{4}-m X^{3} Y-6 X^{2} Y^{2}+m X Y^{3}+Y^{4}=c
$$


for $m, c \in \mathbb{Z}$ with $c \neq 0$. Note that $f_{m}(X)=F_{m}(X, 1)$. The equation $F_{m}(X, Y)=c$ has the following solutions

$$
F_{m}(0, \pm e)=F_{m}( \pm e, 0)=e^{4}, \quad F_{m}(\mp e, \pm e)=F_{m}( \pm e, \pm e)=-4 e^{4} .
$$

We call such solutions $(x, y)$ to $F_{m}(x, y)=c$ with $x y(x+y)(x-y)=0$ the trivial solutions.

For $c \in\{ \pm 1, \pm 4\}$, Lettl-Pethö [LP95] and Chen-Voutier [CV97] gave a complete solution to Thue equation $F_{m}(X, Y)=c$ independently (cf. Section 4).

In LPV99, Lettl-Pethö-Voutier showed that for $m \geq 58$, the only primitive solutions $(x, y) \in \mathbb{Z}^{2}$, i.e. $\operatorname{gcd}(x, y)=1$, to the Thue inequality

$$
\left|F_{m}(x, y)\right| \leq 6 m+7
$$

with $|x| \leq y$ are trivial solutions $(0,1),( \pm 1,1)$ and $( \pm 1,2)$. Note that $F_{m}( \pm 1,2)= \pm 6 m-7$. Wakabayashi Wak07] investigated Thue inequalities $\left|F_{l, m}(x, y)\right| \leq k$ with two parameters $l, m$ and $F_{1, m}=F_{m}$.

For $m \in \mathbb{Z}$, we put

$$
L_{m}:=\operatorname{Spl}_{\mathbb{Q}} f_{m}(X) .
$$

We give the following correspondence between integer solutions to $F_{m}(X, Y)=c$ and isomorphism classes of the simplest quartic fields $L_{m}$.

Theorem 1.4. Let $m \in \mathbb{Z} \backslash\{0, \pm 3\}$ and $L_{m}=\operatorname{Spl}_{\mathbb{Q}} f_{m}(X)$. There exists an integer $n \in \mathbb{Z} \backslash\{ \pm m\}$ such that $L_{n}=L_{m}$ if and only if there exists non-trivial solution $(x, y) \in \mathbb{Z}^{2}$, i.e. $x y(x+y)(x-y) \neq 0$, to the quartic Thue equation

$$
F_{m}(x, y)=c
$$

where $c$ is a divisor of $4\left(m^{2}+16\right)$. Moreover integers $m, n$ and solutions $(x, y) \in \mathbb{Z}^{2}$ to $(*)$ can be chosen to satisfy the equation

$$
N=m+\frac{\left(m^{2}+16\right) x y(x+y)(x-y)}{F_{m}(x, y)}
$$

where either $N=n$ or $N=-n$, and the equation (**) occurs for only one of $N=n$ and $N=-n$.

The assumption $m \neq 0, \pm 3$ ensures that $f_{m}(X)$ is irreducible over $\mathbb{Q}, \operatorname{Gal}_{\mathbb{Q}} f_{m}(X) \cong C_{4}$ and the equality $(* *)$ holds for only one of $N=n$ and $N=-n$. This phenomenon comes from the group theoretical reason (see Section 2). Indeed, in the case of $m= \pm 3$, $f_{ \pm 3}(X)=\left(X^{2} \pm X-1\right)\left(X^{2} \mp 4 X-1\right)$ and the equation $(* *)$ occurs for both of $N=3$ and $N=-3$. Thus non-trivial solutions $(x, y) \in \mathbb{Z}^{2}$ to $(*)$ which satisfy $(* *)$ for $N=-m$ exist (cf. Theorem 1.1). Hence the assumption $m \neq 0, \pm 3$ also ensures that a non-trivial solution $(x, y)$ to $(*)$ corresponds $N \in \mathbb{Z} \backslash\{ \pm m\}$ via $(* *)$.

If there exists an integer $n \in \mathbb{Z} \backslash\{ \pm m\}$ such that $L_{n}=L_{m}$, then we may choose a primitive solution $(x, y) \in \mathbb{Z}^{2}$ to $(*)$ with $(x, y) \equiv(1,0)(\bmod 2)$. Then four solutions $\pm(x, y), \pm(y,-x)$ to $(*)$ for $c=d$ and four solutions $\pm\left(x^{\prime}, y^{\prime}\right), \pm\left(y^{\prime},-x^{\prime}\right)$ to $(*)$ for $c=-4 d$ are primitive, where $\left(x^{\prime}, y^{\prime}\right)=(x-y, x+y)$ and $d$ is an odd divisor of $m^{2}+16$, and only these eight primitive solutions satisfy $(* *)$ for the same $N$ as in Theorem 1.4 
Corollary 1.5. For $m \in \mathbb{Z} \backslash\{0, \pm 3\}$, let $\mathcal{N}$ be the number of primitive solutions $(x, y) \in \mathbb{Z}^{2}$ with $x y(x+y)(x-y) \neq 0$ to $F_{m}(x, y)=c$ where $c$ is a divisor of $4\left(m^{2}+16\right)$. Then we have

$$
\#\left\{n \in \mathbb{Z} \backslash\{ \pm m\} \mid L_{n}=L_{m}, n>0\right\}=\frac{\mathcal{N}}{8}
$$

where $L_{m}=\operatorname{Spl}_{\mathbb{Q}} f_{m}(X)$. In particular, if there does not exist $n \in \mathbb{Z} \backslash\{ \pm m\}$ with $L_{n}=L_{m}$ then $F_{m}(x, y)=c$ where $c$ is a divisor of $4\left(m^{2}+16\right)$ has only trivial solutions $(x, y) \in \mathbb{Z}^{2}$ with $x y(x+y)(x-y)=0$.

However we do not know non-trivial solutions to $(*)$ with $m \geq 0$ except for $m=1,2$, $3,4,22,103,956$. By Theorem 1.1 we can check that $L_{1}=L_{103}, L_{2}=L_{22}, L_{4}=L_{956}$. Some numerical examples will be given in Sections 6,7 and 8 .

\section{Preliminaries}

In order to prove Theorem 1.1, we recall known results of the resolvent polynomials which are fundamental tools in the computational aspects of Galois theory (cf. Coh93, Coh00, Ade01]). We intend to explain how to get an answer to the field intersection problem of $f_{s}(X)=X^{4}-s X^{3}-6 X^{2}+s X+1$, i.e. for $a, b \in K$ how to determine the intersection of $\operatorname{Spl}_{K} f_{a}(X)$ and $\operatorname{Spl}_{K} f_{b}(X)$. An answer to the field isomorphism problem (Theorem 1.1) may be obtained as the special case of the field intersection problem.

Let $\bar{K}$ be a fixed algebraic closure of a field $K$. Let $f(X):=\prod_{i=1}^{m}\left(X-\alpha_{i}\right) \in K[X]$ be a separable polynomial of degree $m$ with some fixed order of the roots $\alpha_{1}, \ldots, \alpha_{m} \in \bar{K}$. By resolvent polynomials with suitable invariants, we may determine the Galois group of the polynomial $f(X)$ over $K$ as follows.

Let $R:=K\left[x_{1}, \ldots, x_{m}\right]$ be the polynomial ring over $K$ with $m$ variables $x_{1}, \ldots, x_{m}$. For $\Theta \in R$, we take a surjective homomorphism $\omega_{f}: R \rightarrow k\left(\alpha_{1}, \ldots, \alpha_{m}\right), \Theta\left(x_{1}, \ldots, x_{m}\right) \mapsto$ $\Theta\left(\alpha_{1}, \ldots, \alpha_{m}\right)$, which is called the specialization map. The kernel of $\omega_{f}$ is the ideal $I_{f}:=$ $\left\{\Theta \in R \mid \Theta\left(\alpha_{1}, \ldots, \alpha_{m}\right)=0\right\}$ in $R$.

Let $S_{m}$ be the symmetric group of degree $m$. We extend the action of $S_{m}$ on $m$ letters $\{1, \ldots, m\}$ to that on $R$ by $\pi\left(\Theta\left(x_{1}, \ldots, x_{m}\right)\right):=\Theta\left(x_{\pi(1)}, \ldots, x_{\pi(m)}\right)$. We define the Galois group of $f(X)$ over $K$ by $\operatorname{Gal}(f / K):=\left\{\pi \in S_{m} \mid \pi\left(I_{f}\right) \subseteq I_{f}\right\}$. Then the Galois group of the splitting field $\operatorname{Spl}_{K} f(X)$ of $f(X)$ over $K$ is isomorphic to $\operatorname{Gal}(f / K)$. If we take another ordering of roots $\alpha_{\pi(1)}, \ldots, \alpha_{\pi(m)}$ of $f(X)$ for some $\pi \in S_{m}$, the corresponding realization of $\operatorname{Gal}(f / K)$ is conjugate in $S_{m}$. Hence, for arbitrary ordering of the roots of $f(X), \operatorname{Gal}(f / K)$ is determined up to conjugacy in $S_{m}$.

For $H \leq U \leq S_{m}$, an element $\Theta \in R$ is called a $U$-primitive $H$-invariant if $H=\operatorname{Stab}_{U}(\Theta)$ $:=\{\pi \in U \mid \pi(\Theta)=\Theta\}$. For a $U$-primitive $H$-invariant $\Theta$, the polynomial

$$
\mathcal{R P}_{\Theta, U}(X)=\prod_{\bar{\pi} \in U / H}(X-\pi(\Theta)) \in R^{U}[X]
$$

where $\bar{\pi}$ runs through the left cosets of $H$ in $U$, is called the formal $U$-relative $H$-invariant resolvent by $\Theta$. The polynomial

$$
\mathcal{R} \mathcal{P}_{\Theta, U, f}(X):=\omega_{f}\left(\mathcal{R} \mathcal{P}_{\Theta, U}(X)\right)
$$

is called the $U$-relative $H$-invariant resolvent of $f$ by $\Theta$. The following theorem is fundamental in the theory of resolvent polynomials (see e.g. [Ade01, p.95]). 
Theorem 2.1. Let $G=\operatorname{Gal}(f / K), H \leq U \leq S_{m}$ be finite groups with $G \leq U$ and $\Theta$ a $U$ primitive $H$-invariant. Suppose that $\mathcal{R} \mathcal{P}_{\Theta, U, f}(X)=\prod_{i=1}^{l} h_{i}^{e_{i}}(X)$ gives the decomposition of $\mathcal{R} \mathcal{P}_{\Theta, U, f}(X)$ into a product of powers of distinct irreducible polynomials $h_{i}(X),(i=$ $1, \ldots, l)$, in $K[X]$. Then we have a bijection

$$
\begin{aligned}
& G \backslash U / H \quad \longrightarrow \quad\left\{h_{1}^{e_{1}}(X), \ldots, h_{l}^{e_{l}}(X)\right\} \\
& G \pi H \quad \longmapsto \quad h_{\pi}(X)=\prod_{\tau H \subseteq G \pi H}\left(X-\omega_{f}(\tau(\Theta))\right)
\end{aligned}
$$

where the product runs through the left cosets $\tau H$ of $H$ in $U$ contained in $G \pi H$, that is, through $\tau=\pi_{\sigma} \pi$ where $\pi_{\sigma}$ runs a system of representative of the left cosets of $G \cap$ $\pi H \pi^{-1}$; each $h_{\pi}(X)$ is irreducible or a power of an irreducible polynomial with $\operatorname{deg}\left(h_{\pi}(X)\right)$ $=|G \pi H| /|H|=|G| /\left|G \cap \pi H \pi^{-1}\right|$.

Corollary 2.2. If $G \leq \pi H \pi^{-1}$ for some $\pi \in U$ then $\mathcal{R} \mathcal{P}_{\Theta, U, f}(X)$ has a linear factor over $K$. Conversely, if $\mathcal{R} \mathcal{P}_{\Theta, U, f}(X)$ has a non-repeated linear factor over $K$ then there exists $\pi \in U$ such that $G \leq \pi H \pi^{-1}$.

Note that when $\mathcal{R} \mathcal{P}_{\Theta, U, f}(X)$ is not squarefree, there exists a suitable Tschirnhausen transformation $\hat{f}$ of $f$ over $K$ such that $\mathcal{R} \mathcal{P}_{\Theta, U, \hat{f}}(X)$ is squarefree (cf. Gir83], Coh93, Alg. $6.3 .4])$.

We apply Theorem 2.1 to the cyclic quartic case. Let $f^{1}(X) \in K[X]$ and $f^{2}(X) \in K[X]$ be separable quartic polynomials over $K$ respectively.

We put

$$
f(X):=f^{1}(X) f^{2}(X)
$$

and

$$
G_{1}:=\operatorname{Gal}\left(f^{1} / K\right), \quad G_{2}:=\operatorname{Gal}\left(f^{2} / K\right), \quad G:=\operatorname{Gal}(f / K) .
$$

We assume that $G_{1}, G_{2} \leq C_{4}$ and apply Theorem 2.1 to $m=8, f(X)=f^{1}(X) f^{2}(X)$, $U=\langle\sigma\rangle \times\langle\tau\rangle, H=\langle\sigma \tau\rangle$ or $\left\langle\sigma \tau^{3}\right\rangle$ where $\sigma, \tau \in S_{8}$ act on $R=K\left[x_{1}, \ldots, x_{8}\right]$ by

$$
\begin{aligned}
\sigma: x_{1} \mapsto x_{2} \mapsto x_{3} \mapsto x_{4} \mapsto x_{1}, \\
\tau: x_{5} \mapsto x_{6} \mapsto x_{7} \mapsto x_{8} \mapsto x_{5} .
\end{aligned}
$$

We put $U:=\langle\sigma\rangle \times\langle\tau\rangle$. Let $\Theta_{1}$ (resp. $\Theta_{2}$ ) be a $U$-primitive $\langle\sigma \tau\rangle$-invariant (resp. $\left\langle\sigma \tau^{3}\right\rangle$ invariant). Then we have the $U$-relative $\langle\sigma \tau\rangle$-invariant (resp. $\left\langle\sigma \tau^{3}\right\rangle$-invariant) resolvent polynomial of $f(X)=f^{1}(X) f^{2}(X)$ by $\Theta_{1}$ (resp. $\left.\Theta_{2}\right)$ as

$$
\mathcal{R}_{f}^{i}(X):=\mathcal{R P}_{\Theta_{i}, U, f}(X), \quad(i=1,2) .
$$

This kind of resolvent polynomial is also called (absolute) multi-resolvent polynomial (cf. [RV99, [Ren04]).

For a squarefree polynomial $\mathcal{R}(X) \in K[X]$ of degree $l$, we define the decomposition type $\mathrm{DT}(\mathcal{R})$ of $\mathcal{R}(X)$ by the partition of $l$ induced by the degrees of the irreducible factors of $\mathcal{R}(X)$ over $K$. By Theorem 2.1, we get the intersection field $\operatorname{Spl}_{K} f^{1}(X) \cap \operatorname{Spl}_{K} f^{2}(X)$ via the decomposition types $\mathrm{DT}\left(\mathcal{R}_{f}^{1}\right)$ and $\operatorname{DT}\left(\mathcal{R}_{f}^{2}\right)$. 
Theorem 2.3. For $f(X)=f^{1}(X) f^{2}(X) \in K[X]$ with $G_{1}, G_{2} \leq C_{4}$, we assume that $\# G_{1} \geq \# G_{2}$ and both $\mathcal{R}_{f}^{1}(X)$ and $\mathcal{R}_{f}^{2}(X)$ are squarefree. Then the Galois group $G=$ $\operatorname{Gal}(f / K)$ and the intersection field $\operatorname{Spl}_{K} f^{1}(X) \cap \operatorname{Spl}_{K} f^{2}(X)$ are given by the decomposition types $\operatorname{DT}\left(\mathcal{R}_{f}^{1}\right)$ and $\mathrm{DT}\left(\mathcal{R}_{f}^{2}\right)$ as on Table 1 .

Table 1

\begin{tabular}{|c|c|c|c|c|c|}
\hline$G_{1}$ & $G_{2}$ & $G$ & & $\overline{\mathrm{DT}}\left(\mathcal{R}_{f}^{1}\right)$ & $\overline{\mathrm{DT}}\left(\mathcal{R}_{f}^{2}\right)$ \\
\hline \multirow{7}{*}{$C_{4}$} & \multirow{4}{*}{$C_{4}$} & $C_{4} \times C_{4}$ & $L_{1} \cap L_{2}=K$ & 4 & 4 \\
\hline & & $C_{4} \times C_{2}$ & {$\left[L_{1} \cap L_{2}: K\right]=2$} & 2,2 & 2,2 \\
\hline & & \multirow{2}{*}{$C_{4}$} & \multirow{2}{*}{$L_{1}=L_{2}$} & 2,2 & $1,1,1,1$ \\
\hline & & & & $1,1,1,1$ & 2,2 \\
\hline & \multirow{2}{*}{$C_{2}$} & $C_{4} \times C_{2}$ & $L_{1} \cap L_{2}=K$ & 4 & 4 \\
\hline & & $C_{4}$ & $L_{1} \supset L_{2}$ & 4 & 4 \\
\hline & $\{1\}$ & $C_{4}$ & $L_{1} \supset L_{2}=K$ & 4 & 4 \\
\hline \multirow{3}{*}{$C_{2}$} & \multirow{2}{*}{$C_{2}$} & $C_{2} \times C_{2}$ & $L_{1} \cap L_{2}=K$ & 2,2 & 2,2 \\
\hline & & $C_{2}$ & $L_{1}=L_{2}$ & $1,1,1,1$ & $1,1,1,1$ \\
\hline & $\{1\}$ & $C_{2}$ & $L_{1} \supset L_{2}$ & 2,2 & 2,2 \\
\hline$\{1\}$ & $\{1\}$ & $\{1\}$ & $L_{1}=L_{2}=K$ & $1,1,1,1$ & $1,1,1,1$ \\
\hline
\end{tabular}

We checked the decomposition types $\operatorname{DT}\left(\mathcal{R}_{f}^{i}\right),(i=1,2)$, on Table 1 by GAP GAP].

Now we get an answer to the field isomorphism problem of

$$
f_{s}(X)=X^{4}-s X^{3}-6 X^{2}+s X+1
$$

via multi-resolvent polynomials $\mathcal{R}_{f_{a, b}}^{i}(X):=\mathcal{R} \mathcal{P}_{\Theta_{i},\langle\sigma\rangle \times\langle\tau\rangle, f_{a, b}},(i=1,2)$, where

$$
f_{a, b}(X):=f_{a}(X) f_{b}(X)
$$

as the special case of Theorem 2.3. Note that $\operatorname{disc}\left(f_{s}(X)\right)=4\left(s^{2}+16\right)^{3}$.

Theorem 2.4. For $a, b \in K$ with $\left(a^{2}+16\right)\left(b^{2}+16\right) \neq 0$, we assume that both $\mathcal{R}_{f_{a, b}}^{1}(X)$ and $\mathcal{R}_{f_{a, b}}^{2}(X)$ are squarefree. Then two splitting fields of $f_{a}(X)$ and of $f_{b}(X)$ over $K$ coincide if and only if $\mathcal{R}_{f_{a, b}}^{1}(X)$ or $\mathcal{R}_{f_{a, b}}^{2}(X)$ splits completely into four linear factors over $K$.

This is an analogue of the classical result by Kummer theory. Namely for a field $K$ which contains a primitive fourth root of unity and $a, b \in K$, the splitting fields of $X^{4}-a$ and of $X^{4}-b$ over $K$ coincide if and only if $X^{4}-a b$ or $X^{4}-a b^{3}$ has a linear factor (equivalent to split completely) over $K$. It is remarkable that Theorem 2.4 does not need the assumption that $K$ contains a primitive fourth root of unity.

\section{Proof of Theorem 1.1}

We give an explicit answer to the field intersection problem of the simplest quartic polynomials $f_{s}(X)$ via suitable invariants $\Theta_{1}$ and $\Theta_{2}$. As the special case, we obtain Theorem 1.1 . 
Let $K(z)$ be the rational function field over $K$ and $\sigma$ a $K$-automorphism of $K(z)$ of order four which is defined by

$$
\sigma: z \mapsto \frac{z-1}{z+1} \mapsto-\frac{1}{z} \mapsto-\frac{z+1}{z-1} \mapsto z
$$

We consider the fixed field $K(z)^{\langle\sigma\rangle}$ and the $C_{4}$-extension $K(z) / K(z)^{\langle\sigma\rangle}$. Then we get

$$
\begin{aligned}
f_{s}(X) & =\prod_{x \in \operatorname{Orb}_{\langle\sigma\rangle}(z)}(X-x)=(X-z)\left(X-\frac{z-1}{z+1}\right)\left(X+\frac{1}{z}\right)\left(X+\frac{z+1}{z-1}\right) \\
& =X^{4}-s X^{3}-6 X^{2}+s X+1
\end{aligned}
$$

where

$$
s=\frac{z^{4}-6 z^{2}+1}{z\left(z^{2}-1\right)}=\frac{\left(z^{2}+2 z-1\right)\left(z^{2}-2 z-1\right)}{z(z+1)(z-1)}
$$

as the generating polynomial of the field extension $K(z) / K(z)^{\langle\sigma\rangle}$. It follows that $K(z)^{\langle\sigma\rangle}=$ $K(s)$ and the Galois group of the polynomial $f_{s}(X)$ over $K(s)$ is isomorphic to $C_{4}$.

We also take another rational function field $K(w)$ over $K$ with indeterminate $w, \tau \in$ $\operatorname{Aut}_{K} K(w)$ with

$$
\tau: w \mapsto \frac{w-1}{w+1} \mapsto-\frac{1}{w} \mapsto-\frac{w+1}{w-1} \mapsto w
$$

and $f_{t}(X)=X^{4}-t X^{3}-6 X^{2}+t X+1$ where

$$
t=\frac{w^{4}-6 w^{2}+1}{w\left(w^{2}-1\right)}=\frac{\left(w^{2}+2 w-1\right)\left(w^{2}-2 w-1\right)}{w(w+1)(w-1)}
$$

by the same manner of $K(z), \sigma$ and $f_{s}(X)$.

Put $U:=\langle\sigma\rangle \times\langle\tau\rangle$. Then the field $K(z, w)$ is $\left(C_{4} \times C_{4}\right)$-extension of $K(z, w)^{U}=K(s, t)$.

In order to apply Theorem 2.4 , we should find suitable $U$-primitive $\langle\sigma \tau\rangle$-invariant $\Theta_{1}$ and $U$-primitive $\left\langle\sigma \tau^{3}\right\rangle$-invariant $\Theta_{2}$.

We may find the following two $U$-primitive $\langle\sigma \tau\rangle$-invariants which are candidates to $\Theta_{1}$ :

$$
\begin{aligned}
\Theta_{1} & =\sum_{i=0}^{3}(\sigma \tau)^{i}(z w)=\frac{(w+z)(w z-1)(z w-w-z-1)(z w+w+z-1)}{z w\left(z^{2}-1\right)\left(w^{2}-1\right)}, \text { or } \\
\Theta_{1} & =\prod_{i=0}^{3}(\sigma \tau)^{i}(z+w) \\
& =\frac{\left(z^{2} w^{2}-z w^{2}-z^{2} w+2 z w+w+z+1\right)\left(z^{2} w^{2}+z w^{2}+z^{2} w+2 z w-w-z+1\right)}{z w\left(z^{2}-1\right)\left(w^{2}-1\right)} .
\end{aligned}
$$

However the multi-resolvent polynomial $\mathcal{R}_{f_{a, b}}^{1}(X)=\mathcal{R} \mathcal{P}_{\Theta_{1}, U, f_{a, b}}(X)$ where $f_{a, b}(X)=$ $f_{a}(X) f_{b}(X)$ becomes complicated in the both cases above.

Example 3.1. We present two explicit examples of the multi-resolvent polynomials $\mathcal{R}_{f_{a, b}}^{i}(X)$ $:=\mathcal{R} \mathcal{P}_{\Theta_{i},\langle\sigma\rangle \times\langle\tau\rangle, f_{a, b}}(X)$ for $i=1,2$ where $f_{a, b}(X):=f_{a}(X) f_{b}(X)$. 
(i) In [HM09b], we gave an answer to the field isomorphism problem of $f_{s}(X)$ by taking

$$
\Theta_{1}=\frac{2\left(z^{2}+1\right)\left(w^{2}+1\right)(z w+1)(z-w)}{z w\left(z^{2}-1\right)\left(w^{2}-1\right)}, \quad \Theta_{2}=\frac{2\left(z^{2}+1\right)\left(w^{2}+1\right)(z w-1)(z+w)}{z w\left(z^{2}-1\right)\left(w^{2}-1\right)} .
$$

Then the corresponding multi-resolvent polynomials are given as

$$
\begin{aligned}
& \mathcal{R}_{f_{a, b}}^{1}(X)=X^{4}-\left(a^{2}+16\right)\left(b^{2}+16\right)\left(X^{2}-4(a-b)^{2}\right), \\
& \mathcal{R}_{f_{a, b}}^{2}(X)=X^{4}-\left(a^{2}+16\right)\left(b^{2}+16\right)\left(X^{2}-4(a+b)^{2}\right) .
\end{aligned}
$$

(ii) If we take another $U$-primitive $\langle\sigma \tau\rangle$-invariant $\Theta_{1}$ and $\left\langle\sigma \tau^{3}\right\rangle$-invariant $\Theta_{2}$ as

$$
\Theta_{1}=\frac{2(z w+1)(z-w)}{\left(z^{2}+1\right)\left(w^{2}+1\right)}, \quad \Theta_{2}=\frac{2(z w-1)(z+w)}{\left(z^{2}+1\right)\left(w^{2}+1\right)}
$$

then we get

$$
\mathcal{R}_{f_{a, b}}^{1}(X)=X^{4}-X^{2}+\frac{4(a-b)^{2}}{\left(a^{2}+16\right)\left(b^{2}+16\right)}, \quad \mathcal{R}_{f_{a, b}}^{2}(X)=X^{4}-X^{2}+\frac{4(a+b)^{2}}{\left(a^{2}+16\right)\left(b^{2}+16\right)} .
$$

The multi-resolvent polynomials in Example 3.1 are useful since they are biquadratic, i.e. quadratic polynomial with respect to $X^{2}$. However we do not understand for a fixed $a \in K$ whether there exist infinitely many $b \in K$ such that $\mathcal{R}_{f_{a, b}}^{i}(X),(i=1,2)$, splits completely over $K$ or not. By Theorem 2.4 this question means that for a fixed $a \in K$ whether there exist infinitely many $b \in K$ such that $\operatorname{Spl}_{K} f_{a}(X)=\operatorname{Spl}_{K} f_{b}(X)$.

It follows from [AHK98, Theorem 1.4] that there exist $\langle\sigma \tau\rangle$-invariant $\Theta_{1}$ and $\left\langle\sigma \tau^{3}\right\rangle$ invariant $\Theta_{2}$ such that $K(z, w)=K\left(z, \Theta_{1}\right)=K\left(z, \Theta_{2}\right)$. The following gives such invariants $\Theta_{1}$ and $\Theta_{2}$ which is the key lemma of this paper.

Lemma 3.2. Let $U=\langle\sigma\rangle \times\langle\tau\rangle$. We take

$$
\Theta_{1}:=\frac{z w+1}{-z+w} \quad \text { and } \quad \Theta_{2}:=\frac{z w-1}{z+w} .
$$

Then the following assertions hold:

(i) the element $\Theta_{1}$ is a $U$-primitive $\langle\sigma \tau\rangle$-invariant;

(ii) the element $\Theta_{2}$ is a $U$-primitive $\left\langle\sigma \tau^{3}\right\rangle$-invariant;

(iii) the $U$-orbit of $\Theta_{i}$ is given by the same as $\langle\sigma\rangle$-orbit of $z$;

$$
\operatorname{Orb}_{U}\left(\Theta_{i}\right)=\left\{\Theta_{i}, \frac{\Theta_{i}-1}{\Theta_{i}+1},-\frac{1}{\Theta_{i}},-\frac{\Theta_{i}+1}{\Theta_{i}-1}\right\}, \quad(i=1,2) .
$$

Proof. We can check the assertions by direct computations.

The multi-resolvent polynomials $\mathcal{R}_{f_{a, b}}^{i}(X):=\mathcal{R} \mathcal{P}_{\Theta_{i},\langle\sigma\rangle \times\langle\tau\rangle, f_{a} f_{b}}(X),(i=1,2)$, with respect to

$$
\Theta_{1}=\frac{z w+1}{-z+w} \text { and } \quad \Theta_{2}=\frac{z w-1}{z+w}
$$

as in Lemma 3.2 are given by

$$
\begin{aligned}
& \mathcal{R}_{f_{a, b}}^{1}(X)=f_{A_{1}}(X)=X^{4}-\frac{a b+16}{-a+b} X^{3}-6 X^{2}+\frac{a b+16}{-a+b} X+1, \\
& \mathcal{R}_{f_{a, b}}^{2}(X)=f_{A_{2}}(X)=X^{4}-\frac{a b-16}{a+b} X^{3}-6 X^{2}+\frac{a b-16}{a+b} X+1
\end{aligned}
$$


where

$$
A_{1}=\frac{a b+16}{-a+b} \text { and } A_{2}=\frac{a b-16}{a+b} .
$$

Note that

$$
\operatorname{disc}\left(\mathcal{R}_{f_{a, b}}^{1}(X)\right)=\frac{4\left(a^{2}+16\right)^{3}\left(b^{2}+16\right)^{3}}{(a-b)^{6}}, \quad \operatorname{disc}\left(\mathcal{R}_{f_{a, b}}^{2}(X)\right)=\frac{4\left(a^{2}+16\right)^{3}\left(b^{2}+16\right)^{3}}{(a+b)^{6}} .
$$

By Theorem 2.3, we get the intersection field $\operatorname{Spl}_{K} f_{a}(X) \cap \operatorname{Spl}_{K} f_{b}(X)$ via Table 1 .

Theorem 3.3. Let $\mathcal{R}_{f_{a, b}}^{i}(X),(i=1,2)$, be as in (11). For $a, b \in K$ with $a \neq \pm b$ and $\left(a^{2}+16\right)\left(b^{2}+16\right) \neq 0$, we assume that $\# \operatorname{Gal}_{K} f_{a}(X) \geq \# \mathrm{Gal}_{K} f_{b}(X)$. Then the intersection field $\operatorname{Spl}_{K} f_{a}(X) \cap \operatorname{Spl}_{K} f_{b}(X)$ is given by the decomposition types $\operatorname{DT}\left(\mathcal{R}_{f_{a, b}}^{1}\right)$ and $\mathrm{DT}\left(\mathcal{R}_{f_{a, b}}^{2}\right)$ as on Table 1 in Theorem 2.3 .

Proof of Theorem 1.1. As the special case of Theorem 3.3, we see the conditions (i) and (ii) are equivalent (cf. also Theorem 2.4).

The condition (iii) is just a restatement of (ii). Indeed, we may check that $z \in K$ is a root of $f_{A_{1}}(X)$ (resp. $f_{A_{2}}(X)$ ) if and only if $z$ satisfies the condition (iii) for $B=b$ (resp. $B=-b$ ). Note that if $z$ is a root of $f_{A_{i}}(X)$ then $\frac{z-1}{z+1},-\frac{1}{z},-\frac{z+1}{z-1}$ are also roots of $f_{A_{i}}(X)$ for $i=1,2$. By Table 1 as in Theorem 2.3. if $\operatorname{Spl}_{K} f_{a}(X) \cong C_{4}\left(\operatorname{resp} . C_{2}\right.$ or $\left.\{1\}\right)$ and $f_{A_{i}}(z)$ splits completely then $f_{A_{j}}(X)$ is irreducible (resp. splits completely) over $K$ for $(i, j)=(1,2)$ and $(2,1)$. This completes the proof.

\section{Simplest quartic fields and related Thue equations}

In this section, we treat the case of $K=\mathbb{Q}$ and $a=m \in \mathbb{Z}$ for

$$
f_{a}(X)=f_{m}(X)=X^{4}-m X^{3}-6 X^{2}+m X+1 \in \mathbb{Z}[X] .
$$

We first see

Lemma 4.1. (i) For $m \in \mathbb{Z} \backslash\{0, \pm 3\}, f_{m}(X)$ is irreducible over $\mathbb{Q}$;

(ii) $f_{ \pm 3}(X)=\left(X^{2} \pm X-1\right)\left(X^{2} \mp 4 X-1\right), f_{0}(X)=\left(X^{2}+2 X-1\right)\left(X^{2}-2 X-1\right)$.

Proof. It follows from $\mathrm{Gal}_{\mathbb{Q}(s)} f_{s}(X) \cong C_{4}$ that $\mathrm{Gal}_{\mathbb{Q}} f_{m}(X) \cong C_{4}, C_{2}$ or $\{1\}$ for $m \in \mathbb{Z}$. Thus if $f_{m}(X)$ is reducible over $\mathbb{Q}$ then there exist $a, b, c \in \mathbb{Z}$ such that $f_{m}(X)=\left(X^{2}+\right.$ $a X+c)\left(X^{2}+b X+c\right)$ with $c= \pm 1$. By comparing the coefficients, we see $c=-1$, because if $c=1$ then $(m, a, b)=(0, \pm 2 \sqrt{2}, \mp 2 \sqrt{2})$. Also by comparing the coefficients, if $c=-1$ then $(m, a)=(-b+4 / b,-4 / b) \in \mathbb{Z}^{2}$. Hence $b \in\{ \pm 1, \pm 2, \pm 4\}$. In each case, we have $(m, a, b)=(3,-4,1),(-3,4,-1),(0,-2,2),(0,2,-2),(-3,-1,4),(3,1,-4)$.

For $m \in \mathbb{Z} \backslash\{0, \pm 3\}$, the splitting fields $L_{m}:=\operatorname{Spl}_{\mathbb{Q}} f_{m}(X)$ of $f_{m}(X)$ over $\mathbb{Q}$ are totally real cyclic quartic number fields and called the simplest quartic fields.

We consider the related parametric family of quartic Thue equations

$$
F_{m}(X, Y):=X^{4}-m X^{3} Y-6 X^{2} Y^{2}+m X Y^{3}+Y^{4}=c
$$

for $m, c \in \mathbb{Z}$ with $c \neq 0$. Note that $f_{m}(X)=F_{m}(X, 1)$. 
We may assume that $m \geq 0$ because if $(x, y) \in \mathbb{Z}^{2}$ is a solution to $F_{m}(x, y)=c$ then $(y, x)$ becomes a solution to $F_{-m}(y, x)=c$. The equation $F_{m}(x, y)=c$ has the following solutions

$$
F_{m}(0, \pm e)=F_{m}( \pm e, 0)=e^{4}, \quad F_{m}(\mp e, \pm e)=F_{m}( \pm e, \pm e)=-4 e^{4} .
$$

We call such solutions $(x, y) \in \mathbb{Z}^{2}$ to $F_{m}(x, y)=c$ with $x y(x+y)(x-y)=0$ the trivial solutions.

If $(x, y) \in \mathbb{Z}^{2}$ is a solution to $F_{m}(x, y)=c$ then four pairs $\pm(x, y), \pm(y,-x)$ are also solutions because $F_{m}(X, Y)$ is invariant under the action $X \longmapsto Y \longmapsto-X$ of order four.

For $c \in\{ \pm 1, \pm 4\}$, Lettl-Pethö LP95] and Chen-Voutier [CV97] gave a complete solution to Thue equation $F_{m}(X, Y)=c$ independently. For $c \in\{ \pm 1, \pm 4\}$ and $m \geq 0$, all solutions to $F_{m}(X, Y)=c$ are given by eight trivial solutions

$$
F_{m}(0, \pm 1)=F_{m}( \pm 1,0)=1, \quad F_{m}(\mp 1, \pm 1)=F_{m}( \pm 1, \pm 1)=-4
$$

for arbitrary $m \geq 0$ and additionally

$$
\begin{array}{ll}
F_{1}(\mp 2, \pm 1)=F_{1}( \pm 1, \pm 2)=-1, & F_{1}( \pm 3, \pm 1)=F_{1}(\mp 1, \pm 3)=4, \\
F_{4}(\mp 3, \pm 2)=F_{4}( \pm 2, \pm 3)=1, & F_{4}( \pm 5, \pm 1)=F_{4}(\mp 1, \pm 5)=-4 .
\end{array}
$$

We put

$$
\left(x^{\prime}, y^{\prime}\right):=(x-y, x+y) .
$$

Then if $(x, y) \in \mathbb{Z}^{2}$ is a solution to $F_{m}(x, y)=c$ then $\left(x^{\prime}, y^{\prime}\right) \in \mathbb{Z}^{2}$ is a solution to $F_{m}\left(x^{\prime}, y^{\prime}\right)=-4 c$. Conversely if $\left(x^{\prime}, y^{\prime}\right)$ is a solution to $F_{m}\left(x^{\prime}, y^{\prime}\right)=-4 c$ then $x^{\prime} \equiv y^{\prime}$ $(\bmod 2)$ and $(x, y)=\left(\frac{x^{\prime}+y^{\prime}}{2}, \frac{-x^{\prime}+y^{\prime}}{2}\right) \in \mathbb{Z}^{2}$ is a solution to $F_{m}(x, y)=c$.

In [LPV99, Lettl-Pethö-Voutier showed that for $m \geq 58$, the only primitive solutions $(x, y) \in \mathbb{Z}^{2}$, i.e. $\operatorname{gcd}(x, y)=1$, to the Thue inequality

$$
\left|F_{m}(x, y)\right| \leq 6 m+7
$$

are trivial solutions $\pm(0,1), \pm(1,0), \pm(1,1), \pm(-1,1)$ and non-trivial solutions $\pm(2,1)$, $\pm(-1,2), \pm(-2,1), \pm(1,2)$. We note that $F_{m}( \pm 2,1)=F_{m}(\mp 1,2)=\mp 6 m-7$.

If $(x, y) \in \mathbb{Z}^{2}$ is a primitive solution to $(*)$ then four pairs $\pm(x, y), \pm(y,-x)$ are primitive solutions to $(*)$. We also see the following lemma:

Lemma 4.2. Put $\left(x^{\prime}, y^{\prime}\right):=(x-y, x+y)$. If $(x, y) \in \mathbb{Z}^{2}$ with $(x, y) \equiv(0,1)$ or $(1,0)$ $(\bmod 2)$ is a primitive solution to $F_{m}(x, y)=c$ then $c$ is an odd integer and $\left(x^{\prime}, y^{\prime}\right) \equiv(1,1)$ $(\bmod 2)$ is a primitive solution to $F_{m}\left(x^{\prime}, y^{\prime}\right)=-4 c$. Conversely if $\left(x^{\prime}, y^{\prime}\right) \in \mathbb{Z}^{2}$ with $\left(x^{\prime}, y^{\prime}\right) \equiv(1,1)(\bmod 2)$ is a primitive solution to $F_{m}\left(x^{\prime}, y^{\prime}\right)=d$ then $d=-4 c$ for an odd integer $c$ and $(x, y)=\left(\frac{x^{\prime}+y^{\prime}}{2}, \frac{-x^{\prime}+y^{\prime}}{2}\right) \equiv(0,1)$ or $(1,0)(\bmod 2)$ is a primitive solution to $F_{m}(x, y)=c$.

Proof. Assume that $(x, y) \in \mathbb{Z}^{2}$ with $(x, y) \equiv(0,1)$ or $(1,0)$ ( $\left.\bmod 2\right)$ is a primitive solution to $F_{m}(x, y)=c$. Then $c$ is odd because $F_{m}(0,1)=F_{m}(1,0)=1$. It follows by the definition that $\left(x^{\prime}, y^{\prime}\right) \equiv(1,1)(\bmod 2)$ and $F_{m}\left(x^{\prime}, y^{\prime}\right)=-4 c$. If $\operatorname{gcd}\left(x^{\prime}, y^{\prime}\right)=k^{\prime}>1$ then $k^{\prime}$ is odd and $k^{\prime}$ divides both $x=\frac{x^{\prime}+y^{\prime}}{2}$ and $y=\frac{-x^{\prime}+y^{\prime}}{2}$. This contradicts to $\operatorname{gcd}(x, y)=1$. Hence we have $\operatorname{gcd}\left(x^{\prime}, y^{\prime}\right)=1$.

Conversely we assume that $\left(x^{\prime}, y^{\prime}\right) \in \mathbb{Z}^{2}$ with $\left(x^{\prime}, y^{\prime}\right) \equiv(1,1)(\bmod 2)$ is a primitive solution to $F_{m}\left(x^{\prime}, y^{\prime}\right)=d$. Then $F_{m}(1,1)=-4 \equiv 0(\bmod 4)$ and $\not \equiv 0(\bmod 8)$. Thus 
$d=-4 c$ for an odd integer $c \in \mathbb{Z}$. We also see $F_{m}(x, y)=c$. If $\operatorname{gcd}(x, y)=k>1$ then $k$ divides both $x^{\prime}=x-y$ and $y^{\prime}=x+y$. This contradicts to $\operatorname{gcd}\left(x^{\prime}, y^{\prime}\right)=1$. Therefore we have $\operatorname{gcd}(x, y)=1$. Because if $(x, y) \equiv(1,1)(\bmod 2)$ then $F_{m}(x, y) \equiv 0(\bmod 4)$, we also obtain $(x, y) \equiv(0,1)$ or $(1,0)(\bmod 2)$.

\section{Proof of Theorem 1.4: the correspondence}

The aim of this section is to establish the correspondence between isomorphism classes of the simplest quartic fields $L_{m}$ and non-trivial solutions to quartic Thue equations $(*)$ as follows:

Theorem (Theorem 1.4). Let $m \in \mathbb{Z} \backslash\{0, \pm 3\}$ and $L_{m}=\operatorname{Spl}_{\mathbb{Q}} f_{m}(X)$. Then there exists an integer $n \in \mathbb{Z} \backslash\{ \pm m\}$ such that $L_{m}=L_{n}$ if and only if there exists non-trivial solution $(x, y) \in \mathbb{Z}^{2}$, i.e. $x y(x+y)(x-y) \neq 0$, to the quartic Thue equation

$$
F_{m}(x, y)=x^{4}-m x^{3} y-6 x^{2} y^{2}+m x y^{3}+y^{4}=c
$$

where $c$ is a divisor of $4\left(m^{2}+16\right)$. Moreover integers $m, n$ and solutions $(x, y) \in \mathbb{Z}^{2}$ to $(*)$ can be chosen to satisfy the equation

$$
N=m+\frac{\left(m^{2}+16\right) x y(x+y)(x-y)}{F_{m}(x, y)}
$$

where either $N=n$ or $N=-n$, and the equation (**) occurs for only one of $N=n$ and $N=-n$.

Proof of Theorem 1.4 We use Theorem 1.1 in the case where $K=\mathbb{Q}$.

For $m \in \mathbb{Z} \backslash\{0, \pm 3\}$, we assume that there exists an integer $n \in \mathbb{Z} \backslash\{ \pm m\}$ such that $L_{m}=L_{n}$. By Theorem 1.1 there exists $z \in \mathbb{Q}$ such that

$$
N=m+\frac{\left(m^{2}+16\right) z(z+1)(z-1)}{f_{m}(z)}
$$

where either $N=n$ or $N=-n$. Write $z=x / y$ with $x, y \in \mathbb{Z}$ and $\operatorname{gcd}(x, y)=1$ then we have

$$
N=m+\frac{\left(m^{2}+16\right) x y(x+y)(x-y)}{F_{m}(x, y)} \in \mathbb{Z} .
$$

By the assumption $n \neq \pm m$, we have $x y(x+y)(x-y) \neq 0$.

We will show that $c:=F_{m}(x, y)$ divides $4\left(m^{2}+16\right)$. We make use of a resultant and the Sylvester matrix (cf. [PV00, [SWP08, Section 1.3], see also [Lan78, Theorem 6.1], [Lan83, Chapter 8, Section 5]).

Put $h(z):=\left(m^{2}+16\right) z(z+1)(z-1)$ and $f(z):=F_{m}(z, 1)$. We take the resultant

$$
R_{m}:=\operatorname{Res}_{z}(h(z), f(z))=16\left(m^{2}+16\right)^{4}
$$


of $h(z)$ and $f(z)$ with respect to $z$. We see that $R_{m}$ is also given by

$$
\begin{aligned}
R_{m} & =\left|\begin{array}{lllllll}
m^{2}+16 & 0 & -m^{2}-16 & 0 & 0 & 0 & h(z) z^{3} \\
0 & m^{2}+16 & 0 & -m^{2}-16 & 0 & 0 & h(z) z^{2} \\
0 & 0 & m^{2}+16 & 0 & -m^{2}-16 & 0 & h(z) z \\
0 & 0 & 0 & m^{2}+16 & 0 & -m^{2}-16 & h(z) \\
1 & -m & -6 & m & 1 & 0 & f(z) z^{2} \\
0 & 1 & -m & -6 & m & 1 & f(z) z \\
0 & 0 & 1 & -m & -6 & m & f(z)
\end{array}\right| \\
& =4\left(m^{2}+16\right)^{3}(h(z) p(z)+f(z) q(z))
\end{aligned}
$$

where

$$
p(z)=5 z^{3}-5 m z^{2}-29 z+4 m, \quad q(z)=-\left(m^{2}+16\right)\left(5 z^{2}-4\right) .
$$

Hence we have

$$
h(z) p(z)+f(z) q(z)=4\left(m^{2}+16\right) .
$$

Put

$$
\begin{aligned}
H(x, y) & :=\left(m^{2}+16\right) x y(x+y)(x-y), \\
P(x, y) & :=5 x^{3}-5 m x^{2} y-29 x y^{2}+4 m y^{3}, \\
Q(x, y) & :=-\left(m^{2}+16\right) y\left(5 x^{2}-4 y^{2}\right) .
\end{aligned}
$$

Then it follows from $z=x / y$ that

$$
H(x, y) P(x, y)+F_{m}(x, y) Q(x, y)=4\left(m^{2}+16\right) y^{7} .
$$

Because the cubic forms $F_{m}(X, Y)$ and $H(X, Y)$ are invariants under the action of $\sigma: X \mapsto$ $Y, Y \mapsto-X$, we also get

$$
H(x, y) P(y,-x)+F_{m}(x, y) Q(y,-x)=4\left(m^{2}+16\right)(-x)^{7} .
$$

Hence we have

$$
\begin{aligned}
& \frac{H(x, y) P(x, y)}{F_{m}(x, y)}+Q(x, y)=\frac{4\left(m^{2}+16\right) y^{7}}{F_{m}(x, y)} \in \mathbb{Z}, \\
& \frac{H(x, y) P(y,-x)}{F_{m}(x, y)}+Q(y,-x)=-\frac{4\left(m^{2}+16\right) x^{7}}{F_{m}(x, y)} \in \mathbb{Z} .
\end{aligned}
$$

Since $x$ and $y$ are relatively prime, we conclude that $c=F_{m}(x, y)$ divides $4\left(m^{2}+16\right)$.

Conversely if there exists $(x, y) \in \mathbb{Z}^{2}$ with $x y(x+y)(x-y) \neq 0$ such that $c=F_{m}(x, y)$ divides $4\left(m^{2}+16\right)$ then we can take $N \in \mathbb{Q} \backslash\{m\}$ by

$$
N=m+\frac{\left(m^{2}+16\right) x y(x+y)(x-y)}{F_{m}(x, y)} .
$$

From the assumption $m \in \mathbb{Z} \backslash\{0, \pm 3\}$, we have $\mathrm{Gal}_{\mathbb{Q}} f_{m}(X) \cong C_{4}$. Hence it follows from Theorem 1.1 (i) and (iii) that $N \in \mathbb{Q} \backslash\{ \pm m\}$ and $L_{m}=L_{N}$.

We should show that $N \in \mathbb{Z}$. If $x \not \equiv y(\bmod 2)$ then $c=F_{m}(x, y)$ divides $m^{2}+16$ and hence $N \in \mathbb{Z} \backslash\{ \pm m\}$, because $F_{m}(x, y) \equiv F_{m}(x, y) \equiv 1(\bmod 2)$.

If $x \equiv y(\bmod 2)$ then $c=F_{m}(x, y)$ divides $\left(m^{2}+16\right) x y(x+y)(x-y)$ and hence $N \in \mathbb{Z} \backslash\{ \pm m\}$, because $x y(x+y)(x-y) \equiv 0(\bmod 4)$. 


\section{Primitive solutions}

By the proof of Theorem 1.1 and Theorem 1.4 a non-trivial solution $(x, y) \in \mathbb{Z}^{2}$ to $(*)$ may be obtained as $z=x / y$ with $\operatorname{gcd}(x, y)=1$ where $z \in \mathbb{Q}$ is a root of $f_{A}(X)=$ $X^{4}-A X^{3}-6 X^{2}+A X+1$ for $A=A_{1}$ or $A=A_{2}$ with

$$
A_{1}=\frac{m n+16}{-m+n} \text { and } A_{2}=\frac{m n-16}{m+n}
$$

as in Theorem 1.1 (ii). Hence we now consider only primitive solutions $(x, y) \in \mathbb{Z}^{2}$, i.e. $\operatorname{gcd}(x, y)=1$, to $(*)$.

Lemma 6.1. Let $m \in \mathbb{Z} \backslash\{0, \pm 3\}$ and $L_{m}=\operatorname{Spl}_{\mathbb{Q}} f_{m}(X)$. We assume that there exists $n \in \mathbb{Z} \backslash\{ \pm m\}$ such that $L_{n}=L_{m}$.

(i) We may choose non-trivial primitive solution $(x, y) \in \mathbb{Z}^{2}$ with $(x, y) \equiv(0,1)(\bmod 2)$ to $F_{m}(x, y)=d$ where $d$ is an odd divisor of $m^{2}+16$. Then four pairs $\pm(x, y), \pm(y,-x)$ are primitive solutions to $F_{m}(X, Y)=d$ and four pairs $\pm\left(x^{\prime}, y^{\prime}\right), \pm\left(y^{\prime},-x^{\prime}\right)$ are primitive solutions to $F_{m}(X, Y)=-4 d$ where $\left(x^{\prime}, y^{\prime}\right)=(x-y, x+y)$.

(ii) All primitive solutions to (*) which satisfy (**) for either $N=n$ or $N=-n$ are given by the eight solutions as in (i), and such solutions exist for only one of $N=n$ and $N=-n$.

Proof. By Theorem 1.4 there exists non-trivial solution $\left(x_{0}, y_{0}\right) \in \mathbb{Z}^{2}$ to $F_{m}\left(x_{0}, y_{0}\right)=c$ for a divisor $c$ of $4\left(m^{2}+16\right)$. By Lemma 4.2, we may choose $(x, y) \in \mathbb{Z}^{2}$ with $(x, y) \equiv(0,1)$ or $\equiv(1,0)(\bmod 2)$ to $F_{m}(x, y)=d$ where $d$ is an odd divisor of $m^{2}+16$. Again by Lemma 4.2. eight pairs $\pm(x, y), \pm(y,-x)$ for $d$ and $\pm(x-y, x+y), \pm(x+y,-x+y)$ for $-4 d$ are all primitive solutions. Hence we may assume that $(x, y) \equiv(0,1)(\bmod 2)$.

These eight solutions correspond to the same $N$ as in Theorem 1.4 because

$$
\frac{x y(x+y)(x-y)}{F_{m}(x, y)}
$$

is invariant under the actions of $x \longmapsto y \longmapsto-x$ and $x \longmapsto x-y, y \longmapsto x+y$.

For $z=x / y$, the equation $(* *)$ holds if and only if $f_{A}(z)=0$ where

$$
A=\frac{m N+16}{-m+N}
$$

(see the proof of Theorem 1.1). We see that if $z=x / y$ is a root of $f_{A}(X)$ then other three roots of $f_{A}(X)$ are given by

$$
\frac{z-1}{z+1}=\frac{x-y}{x+y}, \quad-\frac{1}{z}=\frac{y}{-x}, \quad-\frac{z+1}{z-1}=\frac{x+y}{-x+y} .
$$

Hence only the primitive solutions to $(*)$ which satisfy $(* *)$ for $N$ are the eight solutions above.

Corollary (Corollary 1.5). For $m \in \mathbb{Z} \backslash\{0, \pm 3\}$, let $\mathcal{N}$ be the number of primitive solutions $(x, y) \in \mathbb{Z}^{2}$ with $x y(x+y)(x-y) \neq 0$ to $F_{m}(x, y)=c$ where $c$ is a divisor of $4\left(m^{2}+16\right)$. Then we have

$$
\#\left\{n \in \mathbb{Z} \backslash\{ \pm m\} \mid L_{n}=L_{m}, n>0\right\}=\frac{\mathcal{N}}{8}
$$

where $L_{m}=\operatorname{Spl}_{\mathbb{Q}} f_{m}(X)$. In particular, if there does not exist $n \in \mathbb{Z} \backslash\{ \pm m\}$ with $L_{n}=L_{m}$ then $F_{m}(x, y)=c$ where $c$ is a divisor of $4\left(m^{2}+16\right)$ has only trivial solutions $(x, y) \in \mathbb{Z}^{2}$ with $x y(x+y)(x-y)=0$. 
By Theorem 1.1, we obtain

$$
L_{1}=L_{103}, \quad L_{2}=L_{22}, \quad L_{4}=L_{956} .
$$

Hence for $m \in\{1,2,4,22,103,956\}$, we get non-trivial eight primitive solutions to $(*)$ via Theorem 1.4 as on the following table:

Table 2

\begin{tabular}{|c|c|c|c|c|l|}
\hline$m$ & $N$ & $F_{m}(x, y)=c$ & $m^{2}+16$ & $x y(x+y)(x-y)$ & $(x, y)$ \\
\hline 1 & 103 & -1 & 17 & -6 & $\pm(-2,1), \pm(1,2)$ \\
\hline 1 & 103 & 4 & 17 & 24 & $\pm(3,1), \pm(-1,3)$ \\
\hline 2 & -22 & 5 & 20 & -6 & $\pm(-2,1), \pm(1,2)$ \\
\hline 2 & -22 & -20 & 20 & 24 & $\pm(3,1), \pm(-1,3)$ \\
\hline 4 & -956 & 1 & 32 & -30 & $\pm(-3,2), \pm(2,3)$ \\
\hline 4 & -956 & -4 & 32 & 120 & $\pm(5,1), \pm(-1,5)$ \\
\hline 22 & -2 & $125=5^{3}$ & $500=2^{2} 5^{3}$ & -6 & $\pm(-2,1), \pm(1,2)$ \\
\hline 22 & -2 & $-500=-2^{2} 5^{3}$ & $500=2^{2} 5^{3}$ & 24 & $\pm(3,1), \pm(-1,3)$ \\
\hline 103 & 1 & $-625=-5^{4}$ & $10625=5^{4} 17$ & 6 & $\pm(2,1), \pm(-1,2)$ \\
\hline 103 & 1 & $2500=2^{2} 5^{4}$ & $10625=5^{4} 17$ & -24 & $\pm(-3,1), \pm(1,3)$ \\
\hline 956 & -4 & $28561=13^{4}$ & $913952=2^{5} 13^{4}$ & -30 & $\pm(-3,2), \pm(2,3)$ \\
\hline 956 & -4 & $-114244=-2^{2} 13^{4}$ & $913952=2^{5} 13^{4}$ & 120 & $\pm(5,1), \pm(-1,5)$ \\
\hline
\end{tabular}

\section{$7 \quad$ Reducible case}

In the reducible case $m \in\{0, \pm 3\}, f_{m}(X)$ splits as $f_{0}(X)=\left(X^{2}+2 X-1\right)\left(X^{2}-2 X-1\right)$ and $f_{ \pm 3}(X)=\left(X^{2} \pm X-1\right)\left(X^{2} \mp 4 X-1\right)$ over $\mathbb{Q}$, and hence $\operatorname{Spl}_{\mathbb{Q}} f_{0}(X)=\mathbb{Q}(\sqrt{2})$ and $\operatorname{Spl}_{\mathbb{Q}} f_{ \pm 3}(X)=\mathbb{Q}(\sqrt{5})$.

If $m=0$, the trivial solutions correspond to $N= \pm m=0$.

If $m=3$, then the eight trivial solutions $\pm(0,1), \pm(1,0)$ for $c=1$ and $\pm(-1,1), \pm(1,1)$ for $c=-4$ give $N=3$ and non-trivial eight solutions $\pm(2,1), \pm(-1,2)$ for $c=-25$ and $\pm(-3,1), \pm(1,3)$ for $c=100$ give $N=-3$.

Table 3

\begin{tabular}{|c|c|c|c|c|l|}
\hline$m$ & $N$ & $F_{m}(x, y)=c$ & $m^{2}+16$ & $x y(x+y)(x-y)$ & $(x, y)$ \\
\hline 3 & -3 & -25 & 25 & 6 & $\pm(2,1), \pm(-1,2)$ \\
\hline 3 & -3 & 100 & 25 & -24 & $\pm(-3,1), \pm(1,3)$ \\
\hline
\end{tabular}

\section{Computational result}

We do not know non-trivial primitive solutions to $(*)$ for $m \geq 0$ except on Table 2 and Table 3. By the correspondence as in Theorem 1.4, in order to find primitive solutions to (*) we should get $L_{m}=L_{n}$ for some $m \neq \pm n$. In HM09b, Example 5.4], however, we checked with the aid of computer that for integers $0 \leq m<n \leq 10^{5}, L_{m}=L_{n}$ if and only if $(m, n) \in\{(1,103),(2,22),(4,956)\}$. Using Magma [BCP97, we can get the following: 
Theorem 8.1. For $0 \leq m \leq 1000$, all non-trivial primitive solutions $(x, y) \in \mathbb{Z}^{2}$, i.e. $x y(x+y)(x-y) \neq 0$ and $\operatorname{gcd}(x, y)=1$, to $(*)$ are given on Table 2 and Table 3 . In particular, for $0 \leq m \leq 1000$ with $m \notin\{1,2,4,22,103,956\}$ and $n \in \mathbb{Z}, L_{m}=L_{n}$ implies $m=n$ or $m=-n$.

\section{References and Notes}

[Ade01] C. Adelmann, The decomposition of primes in torsion point fields, Lecture Notes in Mathematics, 1761, Springer-Verlag, Berlin, 2001.

[AHK98] H. Ahmad, M. Hajja, M. Kang, Negligibility of projective linear automorphisms, J. Algebra 199, 344-366, 1998.

[BCP97] W. Bosma, J. Cannon, C. Playoust, The Magma algebra system. I. The user language, J. Symbolic Comput. 24, 235-265, 1997.

[Cha96] R. J. Chapman, Automorphism polynomials in cyclic cubic extensions, J. Number Theory 61, 283-291, 1996.

[CV97] J. Chen, P. Voutier, Complete solution of the Diophantine equation $X^{2}+1=d Y^{4}$ and a related family of quartic Thue equations, J. Number Theory 62, 71-99, 1997.

[Coh93] H. Cohen, A course in computational algebraic number theory, Graduate Texts in Mathematics, 138, Springer-Verlag, Berlin, 1993.

[Coh00] H. Cohen, Advanced topics in computational number theory, Graduate Texts in Mathematics, 193, Springer-Verlag, New York, 2000.

[Duq07] S. Duquesne, Elliptic curves associated with simplest quartic fields, J. Théor. Nombres Bordeaux 19, 81-100, 2007.

[Gaa02] I. Gaál, Diophantine equations and power integral bases. New computational methods, Birkhäuser Boston, Inc., Boston, MA, 2002.

[GAP] The GAP Group, GAP — Groups, Algorithms, and Programming, Version 4.4.10; 2007 (http://www.gap-system.org).

[Gir83] K. Girstmair, On the computation of resolvents and Galois groups, Manuscripta Math. 43, 289-307, 1983.

[Gra77] M. N. Gras, Table numérique du nombre de classes et des unités des extensions cycliques réelles de degré 4 de Q, Publ. Math. Fac. Sci. Besançon, fasc 2, 1977/1978.

[HH05] K. Hashimoto, A. Hoshi, Families of cyclic polynomials obtained from geometric generalization of Gaussian period relations, Math. Comp. 74, 1519-1530, 2005.

[HM99] K. Hashimoto, K. Miyake, Inverse Galois problem for dihedral groups, Number theory and its applications (Kyoto, 1997), 165-181, Dev. Math., 2, Kluwer Acad. Publ., Dordrecht, 1999.

[H] A. Hoshi, On correspondence between solutions of a parametric family of cubic Thue equations and isomorphic simplest cubic fields, preprint, arXiv:0810.3374. 
[HM07] A. Hoshi, K. Miyake, Tschirnhausen transformation of a cubic generic polynomial and a 2-dimensional involutive Cremona transformation, Proc. Japan Acad. Ser. A 83, 21-26, 2007.

[HM09a] A. Hoshi, K. Miyake, A geometric framework for the subfield problem of generic polynomials via Tschirnhausen transformation, Number theory and applications, 65-104, Hindustan Book Agency, New Delhi, 2009.

[HM09b] A. Hoshi, K. Miyake, On the field intersection problem of quartic generic polynomials via formal Tschirnhausen transformation, Comment. Math. Univ. St. Pauli $\mathbf{5 8}, 51-86,2009$.

[HM09c] A. Hoshi, K. Miyake, On the field intersection problem of generic polynomials: a survey, RIMS Kôkyûroku Bessatsu B12, 231-247, 2009.

[HM] A. Hoshi, K. Miyake, On the field intersection problem of solvable quintic generic polynomials, to appear in International Journal of Number Theory. Preprint version: arXiv:0804.4875.

[Kid05] M. Kida, Kummer theory for norm algebraic tori, J. Algebra 293, 427-447, 2005.

[Kim04] H. K. Kim Evaluation of zeta functions at $s=-1$ of the simplest quartic fields, Proceedings of the 2003 Nagoya Conference "Yokoi-Chowla Conjecture and Related Problems", 63-73, Saga Univ., Saga, 2004.

[Kom04] T. Komatsu, Arithmetic of Rikuna's generic cyclic polynomial and generalization of Kummer theory, Manuscripta Math. 114, 265-279, 2004.

[Lan78] S. Lang, Elliptic curves: Diophantine analysis, Grundlehren der Mathematischen Wissenschaften, 231, Springer-Verlag, Berlin-New York, 1978.

[Lan83] S. Lang, Fundamentals of Diophantine geometry, Springer-Verlag, New York, 1983.

[Laz91] A. J. Lazarus, On the class number and unit index of simplest quartic fields, Nagoya Math. J. 121, 1-13, 1991.

[LP95] G. Lettl, A. Pethö, Complete solution of a family of quartic Thue equations, Abh. Math. Sem. Univ. Hamburg 65, 365-383, 1995.

[LPV99] G. Lettl, A. Pethö, P. Voutier, Simple families of Thue inequalities, Trans. Amer. Math. Soc. 351, 1871-1894, 1999.

[Lou07] S. R. Louboutin, Efficient computation of root numbers and class numbers of parametrized families of real abelian number fields, Math. Comp. 76, 455-473, 2007.

[Miy99] K. Miyake, Linear fractional transformations and cyclic polynomials, Algebraic number theory (Hapcheon/Saga, 1996), Adv. Stud. Contemp. Math. (Pusan) 1, 137-142, 1999.

[Mor94] P. Morton, Characterizing cyclic cubic extensions by automorphism polynomials, J. Number Theory 49, 183-208, 1994. 
[Oga03] H. Ogawa, Quadratic reduction of multiplicative group and its applications, (Japanese) Algebraic number theory and related topics (Kyoto, 2002), Sūrikaisekikenkyūsho Kōkyūroku No. 1324, 217-224, 2003.

[PV00] D. Poulakis, E. Voskos, On the practical solution of genus zero Diophantine equations, J. Symbolic Comput. 30, 573-582, 2000.

[RV99] N. Rennert and A. Valibouze, Calcul de résolvantes avec les modules de Cauchy, Experiment. Math. 8, 351-366, 1999.

[Ren04] N. Rennert, A parallel multi-modular algorithm for computing Lagrange resolvens, J. Symbolic Comput. 37, 547-556, 2004.

[Rik02] Y. Rikuna, On simple families of cyclic polynomials, Proc. Amer. Math. Soc. 130, 2215-2218, 2002.

[SWP08] J. R. Sendra, F. Winkler, S. Pérez-Díaz, Rational algebraic curves. A computer algebra approach, Algorithms and Computation in Mathematics, 22. Springer, Berlin, 2008.

[Wak07] I. Wakabayashi, Simple families of Thue inequalities, Ann. Sci. Math. Québec 31, 211-232, 2007. 